\title{
Fast Soft Demapping for Underwater Acoustic Communications With Signal Space Diversity
}

\author{
Zi Ye*, Tarak Arbi*, Francois-Xavier Socheleau ${ }^{\dagger}$ and Benoit Geller* \\ *Department U2IS, ENSTA-ParisTech, 91120, Palaiseau, France \\ \{zi.ye, tarak.arbi, benoit.geller\}@ensta-paristech.fr \\ † IMT Atlantique, CNRS Lab-STICC, Brest, France \\ fx.socheleau@imt-atlantique.fr
}

\begin{abstract}
The underwater acoustic channel is time-varying with a particularly limited bandwidth. This makes reliable communications difficult to achieve and limits the information rate. The rotated constellation is an effective technique that saves both bandwidth and energy. It allows to take advantage of the channel diversity and thus to enhance performance of rotated constellations over some fading channels such as underwater acoustic channels. Nevertheless, this solution increases dramatically the complexity at the receiver side. To face this problem, this paper proposes to use a series of rotation angle $\alpha=\arctan (1 / \sqrt{M})$, which introduces several structural properties that can be used to tremendously ease up the soft demapping process. Moreover, this solution achieves almost the same performance as the theoretically quasi-optimum Max-Log demapper with over $80 \%$ of global complexity reduction. This coded modulation has been tested successfully over several acoustic underwater channels.

Index Terms - Rotated and Cyclic Q-delayed (RCQD), Quadrature Amplitude Modulation (QAM), Demodulation complexity, Underwater acoustics communication.
\end{abstract}

\section{INTRODUCTION}

As it is well known, electromagnetic waves are rapidly absorbed by the conductive aquatic environment and can difficultly be used for medium or long distance communications. In particular they are not used in digital communications at "high rate" on kilometric distances (and more). When the transmitted signal is filtered by the acoustic channel, the transfer function is mainly affected by two effects: 1) the limited bandwidth of the underwater acoustic channel decreases with distances, so the attenuation effect increases with distance. 2) the channel has multiple interfering paths, such as direct path, path reflected on the seabed and path reflected on the sea surface; this produces attenuations randomly which are superimposed on the previous attenuation with the distance, and ends up with a limited bandwidth within which some frequencies will be attenuated, and this distorts the received signal.

Rotated constellation is an efficient technique to increase the diversity of the signal and thus the performances and the information rates [1], without degrading neither the spectral efficiency nor the energy consumption. However the real cost of this technique is the high complexity of the demodulation that prevented a wider use of it. Beside, the angle at which the constellation must be rotated is still an open problem.

This work was sponsored by the Bridges project of the European H2020 program.

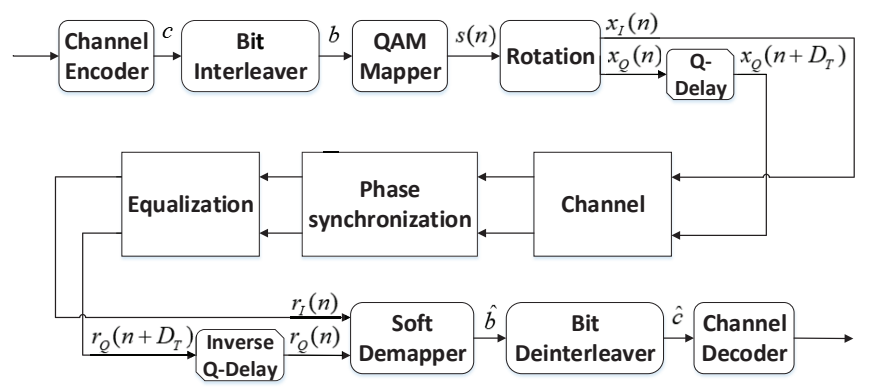

Fig. 1. Underwater acoustics system model with RCQD constellation

Indeed, the angles in [1] are shown to be suboptimal in [2] [5] for both the Rayleigh channel with and without erasure. Thus there is no reason to believe that these angles would be optimal for the underwater channel. The studied rotation angles $\alpha=\arctan (1 / \sqrt{M})$ with $M$ is the modulation order, have interesting properties which has an important effect on the complexity reduction of the demapper [2] [4]. We propose to study this rotated modulation in an underwater environment.

In recent years, numerous physical-layer algorithms and modulation schemes have been proposed. However, comparing faithfully these proposals in an undersea environment is often difficult because the simulation environments are different. In this paper, simulation results were obtained using a realistic simulation platform labeled "watermark" [3]. It includes a library containing the impulse responses of 5 different underwater channels measured in Norway, France and Hawaii.

The outline of this paper is as follows. The system model of the RCQD underwater acoustics system is introduced in Section 2. Our proposed solution including structure properties, demapping algorithm and complexity analysis is presented in Section 3. Section 4 mainly displays the numerical results in the acoustic context and conclusions are made in Section 5.

\section{SYSTEM MODEL}

We now present the system model displayed in Fig. 1.

\section{A. The RCQD M-QAM Signal at the Transmitter Side}

At the transmitter side, information bits are typically coded and interleaved to ensure that bits are transmitted over in- 
dependent fading channels, and then every $\log _{2} M$ bits are mapped into a M-QAM symbol:

$$
s(n)=s_{I}(n)+j s_{Q}(n),
$$

where $s_{I}(n)$ (resp. $\left.s_{Q}(n)\right)$ is the in-phase (resp. quadrature) component at time $n$. The components $s_{m}$ can be expressed in another way such as:

$$
s_{m}=-\sqrt{M}+1+2 p_{m},
$$

where $m$ is in $\{I, Q\}$ and $p_{m}$ takes an integer value in $\{0,1, \ldots, \sqrt{M}-1\}$. This symbol is then rotated with rotation angle $\alpha$ as:

$$
\begin{gathered}
x(n)=x_{I}(n)+j x_{Q}(n)=s(n) \exp (j \alpha), \\
\left\{\begin{array}{l}
x_{I}(n)=s_{I}(n) \cos \alpha-s_{Q}(n) \sin \alpha \\
x_{Q}(n)=s_{I}(n) \sin \alpha+s_{Q}(n) \cos \alpha .
\end{array}\right.
\end{gathered}
$$

From equation (3), we deduce that component $x_{I}(n)$ (resp. $x_{Q}(n)$ ) contain both components $s_{I}(n)$ and $s_{Q}(n)$ which are the complete informations of the unrotated symbol $s(n)$. In order to increase the diversity, the in-phase and quadrature components of symbol $s(n)$ are sent over different (in time) channels. Thanks to the time selectivity of the studied channel, this can be granted by introducing a cyclic delay on the quadrature components, so that the actual symbol transmitted at time n, $y(n)$ can be written as :

$$
y(n)=\beta\left(x_{I}(n)+j x_{Q}\left(n+D_{T}\right)\right),
$$

where $\beta$ is a normalization factor of the QAM constellation and $D_{T}$ is the integer delay.

\section{B. The Soft Demapper at the Receiver Side}

Since the underwater channel is a frequency selective fading channel, we perform on the receiver side front-end, a near optimum [9]- [12] synchronization [13]- [19] and equalization of the acoustic channel [20]- [23]. Such a high performance estimation is mandatory. The received observation $r_{I}(n)$ (resp. $\left.r_{Q}(n)\right)$ of the transmitted component $x_{I}(n)$ (resp. $\left.x_{Q}(n)\right)$ is approximated by:

$$
r_{m}(n)=\beta h_{m}(n) x_{m}(n)+v_{m}(n),
$$

where $m$ is in $\{I, Q\}, h_{I}(n)$ and $h_{Q}(n)$ are the channel attenuation terms, and $v(n)=v_{I}(n)+j v_{Q}(n)$ is a complex Gaussian noise of variance $\sigma^{2}$. In order to simplify the notation, in the sequel, the index $(n)$ is dropped.

For each symbol $x$, the soft demapper computes the loglikelihood (LLR) values for the mapped bits $l^{i}(x) \quad(i=$ $\left.0,1, \ldots, \log _{2} M-1\right)$ as:

$$
\begin{array}{r}
\Upsilon\left(l^{i}(x)\right)=\ln \left(\sum_{x \in \chi_{1}^{i}} \exp \left(\frac{d(r, x)}{\sigma^{2}}\right)\right)- \\
\ln \left(\sum_{x \in \chi_{0}^{i}} \exp \left(\frac{d(r, x)}{\sigma^{2}}\right)\right),
\end{array}
$$

where $\chi_{b}^{i}$ is the set of the constellation points whose i-th bit is $b$, with $b$ is in $\{0,1\}$. If $d(r, x)$ is the squared Euclidean distance between the received symbol and the constellation point $x$ :

$$
d(r, x)=\left|r_{I}-h_{I} x_{I} \beta\right|^{2}+\left|r_{Q}-h_{Q} x_{Q} \beta\right|^{2},
$$

a sub-optimal demapper can be obtained by Max-Log approximation which simplifies the LLR computations:

$$
\Upsilon\left(l^{i}(x)\right)=\frac{1}{\sigma^{2}} \min _{x \in \boldsymbol{\chi}_{1}^{i}}(d(r, x))-\frac{1}{\sigma^{2}} \min _{x \in \boldsymbol{\chi}_{0}^{i}}(d(r, x)) .
$$

(8) implies that for every received bit i, one has to find the closest symbols to the received signal with $\mathrm{i}$-th bit equal to 1 and to 0 . Thus all constellation points should theoretically be considered in the computation of the LLR even with the Max-Log approximation.

\section{PROPOSED SOLUTION}

One can exploit the structure properties brought by the proposed rotation angles $\alpha=\arctan (1 / \sqrt{M})$ in order to process the log-likelihood ratio (LLR) calculus in the integer domain and to locate neighbor constellation points to a received component with a simple manner. How the overall computational complexity can be tremendously reduced is detailed below.

\section{A. Properties of the Rotated Constellation with Rotation Angle $\alpha=\arctan (1 / \sqrt{M})$}

We propose to use the rotation angle $\alpha=\arctan (1 / \sqrt{M})$. $x_{I}$ and $x_{Q}$ in equation (3) can be rewritten as:

$$
\left\{\begin{array}{l}
x_{I}=\left(\sqrt{M} s_{I}-s_{Q}\right) \sin \alpha \\
x_{Q}=\left(\sqrt{M} s_{Q}+s_{I}\right) \sin \alpha
\end{array}\right.
$$

From (2), (9) can be rewritten as:

$$
\left\{\begin{array}{l}
x_{I}=2\left(-\frac{M+1}{2}+\sqrt{M} p_{I}+\left(-p_{Q}+\sqrt{M}-1\right)\right) \sin \alpha \\
x_{Q}=2\left(\left(\sqrt{M} p_{Q}+p_{I}\right)-\frac{M+1}{2}\right) \sin \alpha,
\end{array}\right.
$$

which induces the following properties:

Property 1: The components $x_{I}(n)$ (resp. $x_{Q}(n)$ ) of the rotated symbols are uniformed projected on $I$ (resp. $Q$ ) axes with a constant minimum distance $d_{m}=2 \sin \alpha$ between two consecutive points.

This property can be observed in Fig. 2.

Property 2: There is a one-to-one correspondence between rotated component $x_{I}$ (resp. $x_{Q}$ ) and an integer pair $\left(p_{I}, p_{Q}\right)$. 


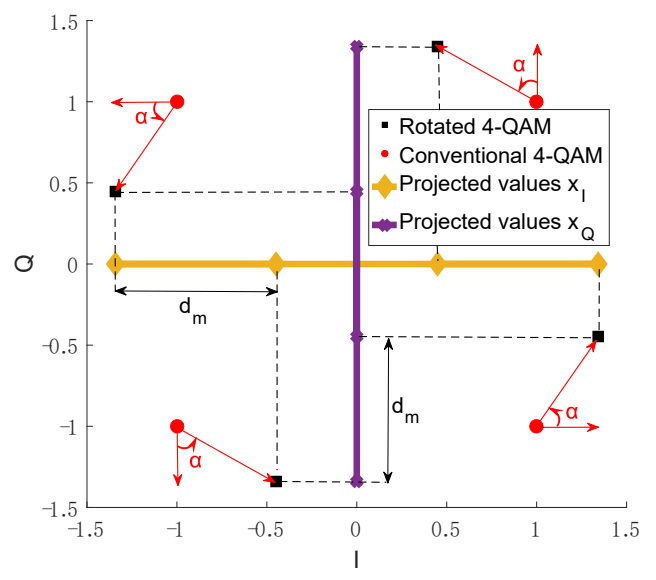

Fig. 2. Uniformly projected RCQD 4-QAM signal

Define an integer pair $\left(T_{I}, T_{Q}\right)$ as:

$$
\left\{\begin{array}{l}
T_{I}=\frac{M-1}{2}+\frac{x_{I}}{2 \sin \alpha} \\
T_{Q}=\frac{M-1}{2}+\frac{x_{Q}}{2 \sin \alpha}
\end{array}\right.
$$

where $T_{m}$ take integer values in $\{0,1, \ldots, \sqrt{M}\}, m \in$ $\{I, Q\}$. One value taken by $T_{I}$ can uniquely determine one integer pair $\left(p_{I}, p_{Q}\right)$ as:

$$
\left\{\begin{array}{l}
p_{I}=\left\lfloor\frac{T_{I}}{\sqrt{M}}\right\rfloor \\
p_{Q}=\sqrt{M}-1-\left(T_{I}-\sqrt{M} p_{I}\right),
\end{array}\right.
$$

Similarly, one value taken by $x_{Q}$ can uniquely determine one integer pair $\left(p_{I}, p_{Q}\right)$ as:

$$
\left\{\begin{array}{l}
p_{I}=\left\lfloor\frac{T_{Q}}{\sqrt{M}}\right\rfloor \\
p_{Q}=-\sqrt{M} p_{Q}+T_{Q}
\end{array}\right.
$$

Property 3: Any $\sqrt{M}$ consecutive integers $T_{I}$ (resp. $T_{Q}$ ) contain every possibly taken integer values $p_{Q}\left(\right.$ resp. $\left.p_{I}\right)$.

\section{B. Proposed Demapper}

On the receiver side, the observation components $r_{m}$ can be transformed as:

$$
\begin{aligned}
R_{m} & =\frac{r_{m}}{2 \beta h_{m} \sin \alpha}+\frac{M-1}{2} \\
& =\frac{x_{m}}{2 \sin \alpha}+\frac{v_{m}}{2 \beta h_{m} \sin \alpha}+\frac{M-1}{2} \\
& =T_{m}+\frac{v_{m}}{2 \beta h_{m} \sin \alpha},
\end{aligned}
$$

where $m \in\{I, Q\}$; therefore:

$$
\left|r_{m}-h_{m} x_{m} \beta\right|^{2}=(2 \beta \sin \alpha)^{2}\left|h_{m}\left(R_{m}-T_{m}\right)\right|^{2},
$$

(7) (resp. (8)) can be rewritten respectively as:

$$
d(R, T)=\left|h_{I}\left(R_{I}-T_{I}\right)\right|^{2}+\left|h_{Q}\left(R_{Q}-T_{Q}\right)\right|^{2},
$$

$$
\begin{aligned}
\Upsilon\left(l^{i}(x)\right) & =\frac{(2 \beta \sin \alpha)^{2}}{\sigma^{2}} \min _{T \in \boldsymbol{T}_{1}^{i}}(d(R, T)) \\
& -\frac{(2 \beta \sin \alpha)^{2}}{\sigma^{2}} \min _{T \in \boldsymbol{T}_{0}^{i}}(d(R, T)),
\end{aligned}
$$

where $\boldsymbol{T}_{0}^{i}$ (resp. $\boldsymbol{T}_{1}^{i}$ ) is the set of the constellation points whose i-th bit is 0 (resp.1).

(17) indicates that computing $\log _{2} M$ LLR values requires $2 \log _{2} M$ minimal distance terms.

Let $T_{o p t}=\left(T_{I, \text { opt }}, T_{Q, \text { opt }}\right)$ be the global optimum point defined as the nearest constellation point to the received observation, and define $T_{\text {comp }}^{i}=\left(T_{I, \text { comp }}^{i}, T_{Q, \text { comp }}^{i}\right)$ as the closest point to the received signal with i-th bit complementary to the i-th bit of $T_{o p t}$. Equation (17) can be rewritten as:

$$
\Upsilon\left(l^{i}(x)\right)=\left\{\begin{array}{l}
\frac{(2 \beta \sin \alpha)^{2}}{\sigma^{2}}\left(d\left(R, T_{\text {opt }}\right)-d\left(R, T_{\text {comp }}^{i}\right)\right), l^{i}\left(T_{\text {opt }}\right)=1, \\
\frac{(2 \beta \sin \alpha)^{2}}{\sigma^{2}}\left(d\left(R, T_{\text {comp }}^{i}\right)-d\left(R, T_{\text {opt }}\right)\right), l^{i}\left(T_{\text {opt }}\right)=0 .
\end{array}\right.
$$

To compute the LLR values (18) one needs to obtain the global optimum and then to search for the $\log _{2} M$ complementary points.

\section{Global Optimum Point $T_{\text {opt }}$}

The nearest point $T_{m}$ to the observation $R_{m}$, where $m$ is in $\{I, Q\}$, can be found equal to:

$$
\widetilde{T}= \begin{cases}0 & \text { if } R_{m}<0 \\ \operatorname{round}\left(R_{m}\right) & \text { if } 0 \leqslant R_{m}<M-1 \\ M-1 & \text { if } R_{m} \geqslant M-1\end{cases}
$$

Each observation $R_{m}$ leads to one local nearest point $\widetilde{T}_{m}$, if $\widetilde{T}_{I}$ and $\widetilde{T}_{Q}$ lead to the same pair $\left(p_{I}, p_{Q}\right)$, then the global optimum point is found. If not, one may increase the number of candidate points by searching points centered around $R_{I}$ and $R_{Q}$. The integer radius of the searching region is $d . \widetilde{T}_{m}$ can be rewritten as:

$\widetilde{\boldsymbol{T}}_{\boldsymbol{m}}= \begin{cases}\{0,1, \ldots, 2 d-1\} & \text { if } R_{m}<d, \\ \{M-2 d, \ldots, M-1\} & \text { if } R_{m} \geqslant M-d, \\ \left\{\left\lfloor R_{m}\right\rfloor-d+1, \ldots,\left\lfloor R_{m}\right\rfloor+d\right\} & \text { otherwise. }\end{cases}$

$\widetilde{\boldsymbol{T}}_{\boldsymbol{m}}$ contains exactly $2 d$ candidate points and each point in $\widetilde{\boldsymbol{T}}_{\boldsymbol{m}}$ uniquely determines a constellation point $\left(x_{I}, x_{Q}\right)$.

\section{Complementary Point $T_{\text {comp }}^{i}$}

According to property 3 , the region $\widetilde{\boldsymbol{T}}_{\boldsymbol{I}}$ (resp. $\widetilde{\boldsymbol{T}}_{\boldsymbol{Q}}$ ) centered around $R_{I}$ (resp. $R_{Q}$ ) with radius $d=\sqrt{M} / 2$ contains all possible values for $p_{Q}$ (resp. $p_{I}$ ), so $\widetilde{\boldsymbol{T}}_{\boldsymbol{I}}$ and $\widetilde{\boldsymbol{T}}_{\boldsymbol{Q}}$ guarantee to find the complementary point $T_{\text {comp }}^{i}$ for any index bit $i$. 


\section{Summary of the Proposed Demapping Algorithm}

We now briefly overview the 4 steps of the proposed demapping algorithm:

1) Using (14), $r_{I}$ and $r_{Q}$ are transformed into $R_{I}$ and $R_{Q}$.

2) Obtain two regions centered around $R_{I}$ and $R_{Q}$ by (20) with $d=\sqrt{M} / 2$, then find the global optimum point within $4 d$ points by calculating the minimum distance from $R$.

3) For each bit, all the points with i-th bit complementary to the i-th bit of $T_{o p t}$ are selected among $4 d$ points, then calculate the minimum distance.

4) Compute the LLR values for each bit by using (18) since we have obtained the Euclidean distances for $T_{\text {opt }}$ and $T_{\text {comp }}^{i}$ in the previous two steps.

\section{Complexity Analysis}

We now display the complexity of the proposed algorithm based on Real Comparisons (RC), Real Sums (RS), Real Multiplications (RM), Real Inversions (RI) and Candidate Points (CP).

\section{At the transmitter side:}

A rotation as in equation (3) with a classical angle of rotation requires 4 RMs and 2 RSs. With $\alpha=\arctan (1 / \sqrt{M})$ however, we get $x_{I}$ and $x_{Q}$ with equation (9); the multiplication by a factor $\sqrt{M}$ can be implemented by $\left(\log _{2} M\right) / 2$ left-shifts. So a rotation operation requires 2 left-shifts, 2 RMs and 2 RSs.

At the receiver side:

- In Step 1, the transform equation (14) needs globally 2 RSs, 2 RMs and 2 RIs.

- In Step 2, choosing the region $\widetilde{\boldsymbol{T}}_{\boldsymbol{m}}$ for $R_{I}$ and $R_{Q}$ requires 4 RCs. Each term $d\left(R, T_{x}\right)=\left|h_{I}\left(R_{I}-T_{I, x}\right)\right|^{2}+$ $\left|h_{Q}\left(R_{Q}-T_{Q, x}\right)\right|^{2}$ requires 4 RMs and 3 RSs. So globally, $2 \sqrt{M}$ distance terms need $8 \sqrt{M}$ RMs and $6 \sqrt{M}$ RSs and to compare $2 \sqrt{M}$ distance terms in order to find the minimum distance needs $(2 \sqrt{M}-1)$ RCs. So step 2 totally requires $8 \sqrt{M}$ RMs, $6 \sqrt{M}$ RSs and $(2 \sqrt{M}+3)$ RCs.

- In Step 3, we need to obtain 1 minimum distance for each bit, which needs $2(\sqrt{M}-1)$ RCs, so a total of $\log _{2} M$ complementary bits require $2(\sqrt{M}-1) \log _{2} M$ RCs.

- In Step $4, \log _{2} M$ bits of one symbol require $\log _{2} M$ RMs and $\log _{2} M$ RSs.

Summing the complexity of the previous four steps, we find that the total complexity of calculating LLR values (18) of one symbol equals to $2 \sqrt{M} \mathrm{CPs},\left(8 \sqrt{M}+\log _{2} M+2\right)$ RMs, $\left(5+(2 \sqrt{M}-2)\left(1+\log _{2} M\right)\right)$ RCs, 2 RIs and $\left(6 \sqrt{M}+\log _{2} M+2\right)$ RSs.

\section{NUMERICAL RESULTS}

\section{A. WATERMARK}

Several physical layer algorithms and modulation techniques have been proposed in recent years. However, it is
TABLE I

THE MEASUREMENT PARAMETERS.

\begin{tabular}{|c|c|c|}
\hline Parameters & KAU2 & NOF1 \\
\hline Time of the year & July & June \\
\hline Water depth & $100 \mathrm{~m}$ & $80 \mathrm{~m}$ \\
\hline -3dB freq. band & $4-8 \mathrm{KHz}$ & $10-18 \mathrm{KHz}$ \\
\hline Duration & $32.9 \mathrm{~s}$ & $32.9 \mathrm{~s}$ \\
\hline Type & SIMO & SISO \\
\hline Hydrophones & 16 & 1 \\
\hline
\end{tabular}
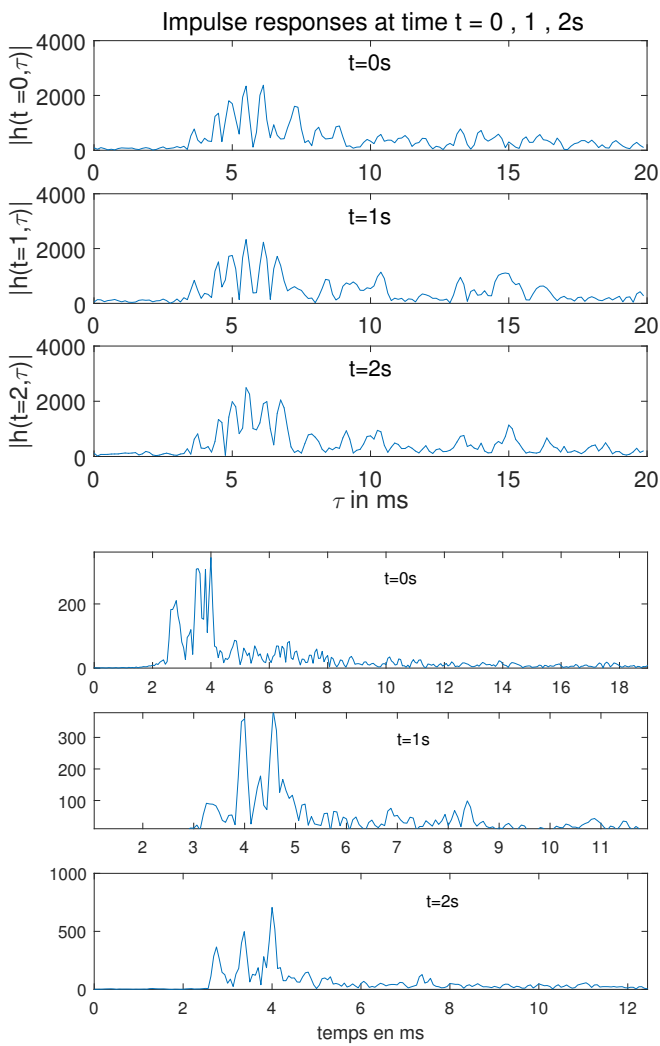

Fig. 3. Impulse responses at times $t=0,1$ and $2 \mathrm{~s}$ of KAU2(up) and NOF1(bottom)

difficult to compare the performance of these proposals objectively because the simulation conditions are different. WATERMARK is a Matlab platform which includes a library containing impulse responses from 5 actual underwater acoustic channels. Simulation results were obtained over two submarine channels (a submarine channel measured off the western side of Kauai, HI, USA, noted KAU2 and a submarine channel from Norway-Oslofjorden noted NOF1). The measurement parameters of the channels are summarized in Table I.

Fig. 3 shows the impulse responses obtained at times $t=0,1,2 s$ of KAU2 and NOF1. Fig. 3 points out that the considered channel is frequency and time selective.

\section{B. Demapper Complexity Comparison}

The complexity cost function of different demapping methods for demmaping one received rotated symbol with rotation 


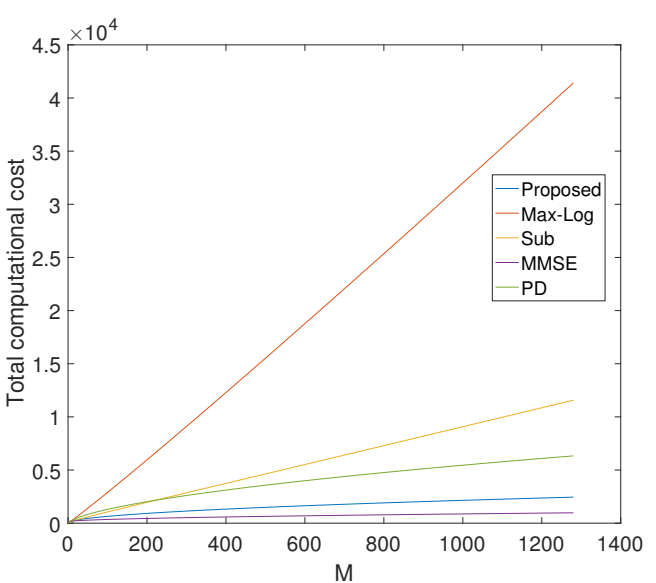

Fig. 4. Complexity comparison of several demappers

angle $\alpha$ are compared in Fig. 4, including the proposed method, the full-complexity Max-Log method, the Sub-Region method [6], the MMSE method [7] and PD-DEM method [8]. We assume that the cost of RC, RS and RM or RI are one, two and four respectively. It can be observed that the MMSE demapper has the lowest complexity among the considered demappers. Nevertheless, as it is shown later in the paper, the BER performance of the MMSE demapper is rather poor. It can be observed as well that the proposed demapper has the lowest complexity among all considered 2 dimensional demappers.

\section{Performance comparison}

Fig. 5 shows the performances in Bit Error Rate (BER) of the proposed angle and rotation angle $\alpha_{1}=\arctan ((1-$ $\sqrt{5}) / 2$ ) which is often considered as asymptotically optimal [1], and the conventional QPSK constellation on KAU2 and NOF1 channels. In this simulation, we consider a $5 / 6$ rate Turbo code, the BER curves are estimated through Monte Carlo simulations; each simulated packet contains 512 bits (64 bytes). Fig. 5 shows that the performances of the proposed method exceed those of the conventional QPSK signals by approximately $2 \mathrm{~dB}$ and exceed those of the rotated signals with angle $\alpha_{1}$ by $0.75 \mathrm{~dB}$ for both channels.

Fig. 6 shows that for the uniformly projected 4-QAM, the proposed demapper reaches the closest performance to the optimal one and performs better than all other studied demappers.

\section{CONClusion}

We studied for several $M$-QAM constellations a set of rotation angles $\alpha=\arctan (1 / \sqrt{M})$. These rotation angles allow a low complexity demapper that gives almost the same performance as the optimal algorithm Max-Log. Thanks to the good performance of the proposed angles and the simplicity of implementation of the proposed demapper, the spectral
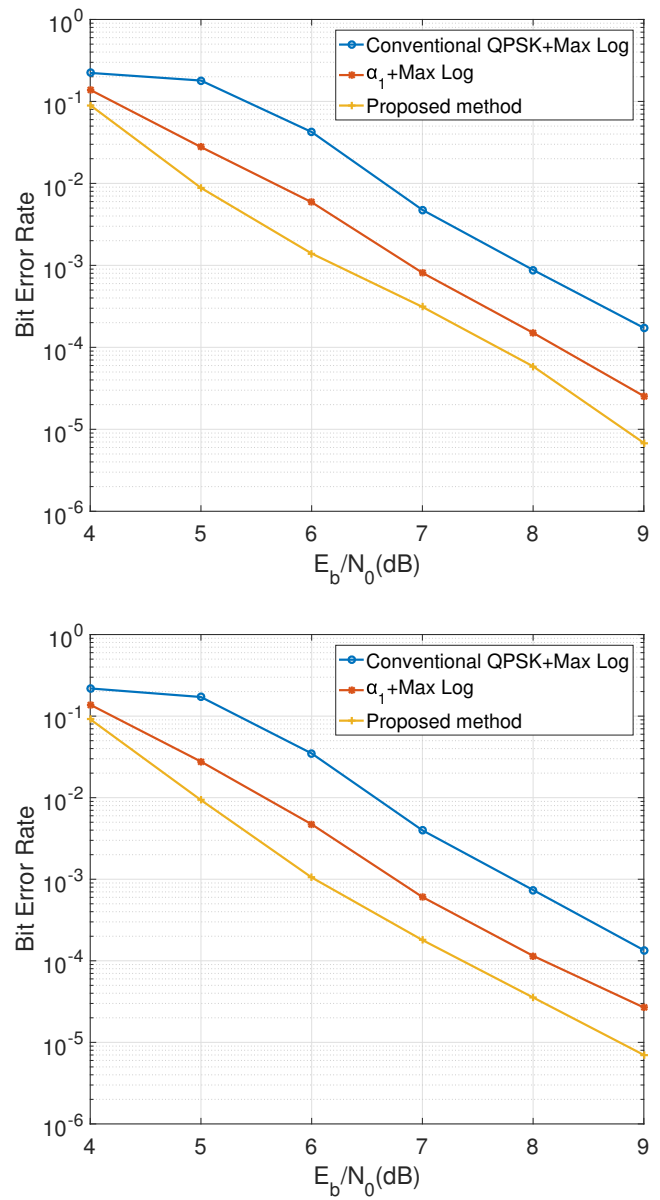

Fig. 5. BER comparison between the proposed method and $\alpha_{1}$ and conventional QPSK signals on NOF1 (top) and KAU2 (bottom) channels

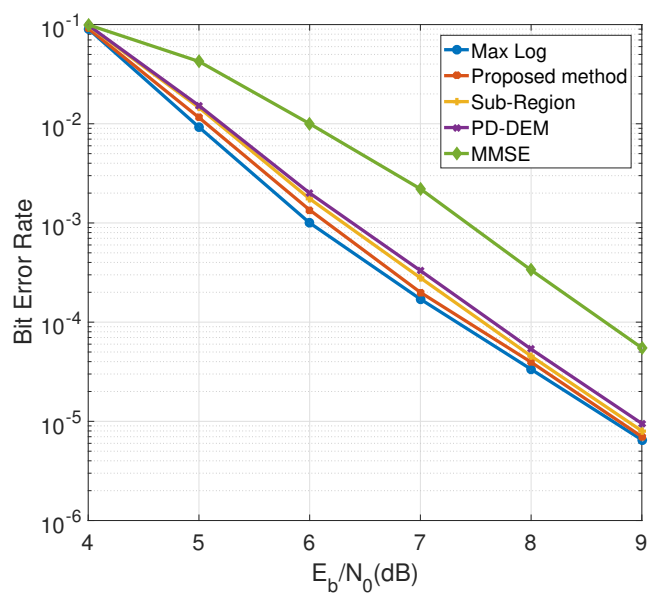

Fig. 6. BER performance of several algorithms for uniformly projected RCQD 4-QAM signal on KAU2 
efficiency and the energy consumption of the underwater communication system can be enhanced. It is important to note that the inherent signal diversity explored without bandwidth expansion could in turn save even more bandwidth by allowing the use of a higher rate channel code [24]- [27].

\section{REFERENCES}

[1] J. Boutros, and E. Viterbo, "Signal space diversity: a power- and bandwidth-efficient diversity technique for the Rayleigh fading channel,' IEEE Trans. on Inform. Theory, 44 (4):1453-1467, July 1998.

[2] T. Arbi, B. Geller, J. Yang, C. Abdel Nour, O. Rioul, "Uniformly Projected RCQD QAM: A Low-Complexity Signal Space Diversity Solution Over Fading Channels With or Without Erasures," IEEE Trans. on Broadcasting, DOI 10.1109/TBC.2018.2811618, IEEE early access, April 2018.

[3] Paul A. van Walree, François-Xavier Socheleau, Roald Otnes, and Trond Jenserud, "The Watermark Benchmark for Underwater Acoustic Modulation Schemes," IEEE Journal of Oceanic Engineering, pp. 10071018 Volume: 42, Issue: 4, Oct. 2017.

[4] J. Yang, K. Wan, B. Geller, C.A. Nour, O. Rioul, C. Douillard, "A LowComplexity 2D Signal Space Diversity Solution for Future Broadcasting Systems," Proc. ICC 2015, June 2015.

[5] J. Yang, M. Li, C.A. Nour, C. Douillard, B. Geller, "Max Log Demapper Architecture Design for DVB T2 Rotated QAM Constellations," Proc. IEEE SIPS, Hangzhou, October 2015.

[6] M. Li, C. Abdel Nour, C. Jego, and C. Douillard, "Design of rotated QAM mapper/demapper for the DVB-T2 standard," Proc. IEEE work. on Sig. Proc. Sys. (SiPS), 2009, pp. 018-023, Oct. 2009.

[7] K. Kim, K. Bae, and H. Yang, "One-dimensional soft-demapping using decorrelation with interference cancellation for rotated QAM constellation," Proc. 2012 IEEE Con. Comm. and Net. Conference (CCNC), pp. 787-791, Jan. 2012.

[8] S. Tomasin, and M. Butussi, "Low complexity demapping of rotated and cyclic Q delayed constellation for DVB-T2," IEEE Wireless Comm. Letters, 1(2):81-84, April 2012.

[9] S. Bay, B. Geller, A. Renaux, J.P. Barbot, J.M. Brossier, "On the Hybrid Cramer-Rao bound and its Application to Dynamical Phase Estimation, IEEE Signal Processing letters, vol. 15, pp. 453-456, 2008.

[10] S. Bay, C. Herzet, J.M. Brossier, J.P. Barbot, B. Geller, "Analytic and Asymptotic Analysis of Bayesian Cramer-Rao Bound for Dynamical Phase Offset Estimation", IEEE Transactions on Signal Processing, vol 56, no 1, pp. 61-70, Jan. 2008.

[11] J. Yang, B. Geller, and S. Bay, "Bayesian and Hybrid Cramr-Rao Bounds for the Carrier Recovery under Dynamic Phase Uncertain Channels", IEEE Transactions on Signal Processing, vol. 59, no 2, pp. 667-680, Feb. 2011.

[12] I.Nasr, L. Najjar Atallah, B. Geller, S. Cherif, "CRB Derivation and New Code-Aided Timing Recovery Technique for QAM Modulated Signals", Proc. of ICC 2015, June 2015.

[13] U. Mengali and A. N.D'Andrea, "Synchronization Techniques for Digital Receivers", Plenum Press, 1997.

[14] I. Nasr, L. Najjar, S. Cherif, B. Geller, J. Yang, "A Soft Maximum Likelihood Technique for Time Delay Recovery," Proc. of IEEE COMNET, Tunis, Mars 2014.

[15] J. Yang, B. Geller, C. Herzet, and J.M. Brossier, "Smoothing PLLs for QAM Dynamical Phase Estimation”, Proc. IEEE ICC'09, June 2009.

[16] I. Nasr, B. Geller, L. N. Atallah, S. Cherif, "Performance Study of a New Near Maximum Likelihood Code-Aided Timing Recovery Technique", IEEE Transactions on Signal Processing, vol. 64, pp. 799-811, Feb. 2016.

[17] I. Nasr, L. Najjar Atallah, S. Cherif, B. Geller, "Near MAP Dynamical Delay Estimator and Bayesian CRB for Coded QAM Signals", IEEE Transactions on Wireless Communications, Volume: 17(1), pp. 636-651, Jan. 2018.

[18] F. Gardner, "Demodulator reference recovery techniques suited for digital implementation", 1988, available on-line.

[19] J. Yang and B. Geller, "Near-optimum Low-Complexity Smoothing Loops for Dynamical Phase Estimation", IEEE Transactions on Signal Processing, vol. 57, no 9, pp. 3704-3711, Sept. 2009.
[20] B. Geller, V. Capellano, J.M. Brossier, A. Essebbar, G. Jourdain, "Equalizer for Video Rate Transmission in Multipath Underwater Communications," IEEE Journal of Oceanic Engineering, vol. 21, no 2, pp 150-156, April 1996.

[21] B. Geller, V. Capellano, J.M. Brossier, "Equalizer for High Rate Transmission", Proc. IEEE OCEANS, Sept. 1994.

[22] B. Geller, V. Capellano, G. Jourdain, "Equalizer for Real Time High Rate Transmission in Underwater Communications", Proc. of IEEE ICASSP, pp 302-305, May 1995.

[23] C. Laot, A. Glavieux, J. Labat, "Turbo equalization: adaptive equalization and channel decoding jointly optimized", IEEE Journal on Selected Areas in Communications, vol. 19 (9), pp. 1744-1752, Sept. 2001.

[24] B. Geller, I. Diatta, J.P. Barbot, C. Vanstraceele, F. Rambeau, "Block Turbo Codes : From Architecture to Application", Proc. of IEEE ISIT, Seattle, July 2006.

[25] C. Vanstraceele, B. Geller, J.P. Barbot, J.M. Brossier, "Block Turbo Codes for Multicarrier Local Loop Transmission", Proc. of IEEE VTC, pp 1773-1775, Vancouver Oct. 2002.

[26] S. Lin and D. J. Costello, "Error Control Coding", Prentice Hall, 2nd ed, 2004.

[27] I. Diatta, D. De Geest, B. Geller, "Reed Solomon Turbo Codes for High Data Rate Transmission", Proceedings of IEEE VTC, pp. 1023-1027, Milan, May 2004. 